Georgian Mathematical Journal

Volume 13 (2006), Number 1, 35-53

\title{
SOLUTION OF A NONCLASSICAL PROBLEM OF OSCILLATION OF TWO-COMPONENT MIXTURES
}

\author{
LEVAN GIORGASHVILI AND KETEVAN SKHVITARIDZE
}

\begin{abstract}
A general representation of solutions by six metaharmonic functions is obtained for a system of homogeneous equations of oscillation of two-component mixtures. The boundary value problem of oscillation of twocomponent mixtures is investigated when the normal components of partial displacement vectors and the tangent components of partial rotation vectors are given on the boundary. Uniqueness theorems of the considered problem are proved. Solutions are obtained in terms of absolutely and uniformly convergent series.
\end{abstract}

2000 Mathematics Subject Classification: 35J55, 74H420, 74H25.

Key words and phrases: Elasticity theory, mixture theory, continual theory of mixtures, mataharmonic function, radiation condition.

\section{INTRODUCTION}

In the early 60 s of the last century, C. Truesdell and R. Toupin formulated in [20] the fundamental mechanical principles of a new model of a deformable elastic medium with complex inner structure and thereby laid the foundation for the continual theory of elastic mixtures. In subsequent years this theory was generalized and developed in different directions. Based on kinematic and thermodynamic principles, theories were created for two- and many-component mixtures of such as fluid-fluid (Crochet and Naghdi [5], Atkin [2], Green and Naghdi [9], [10], Green and Steel [8]), fluid-solid body (Crochet and Naghdi [5], Atkin [2], Green and Naghdi [9], [10], Green and Steel [8] and solid body-solid body (Crochet and Naghdi [5], Atkin [2], Green and Naghdi [9], [10], Green and Steel [8], Khoroshun and Soltanov [12], Hill [11]).

In Natroshvili, Dzhagmaidze and Svanadze [17], static and dynamic problems of the linear theory of a mixture of two isotropic elastic components are investigated by the method of a potential and singular integral equations. Atkin, Chadwick and Steel [3] and Knops and Steel [13] deal with uniqueness theorems for various linearized dynamic problems of the theory of anisotropic mixtures.

Questions as to the existence and uniqueness of weak solutions of mixed static linear problems for mixtures of two nonhomogeneous anisotropic elastic components were considered in Aron [1] and Borrelli and Patria [4]; in the former work, the problem was studied by the method of functional analysis, while in the latter by the variational method. In Khoroshun and Soltanov's monograph [12], along with theoretical questions, quite interesting concrete problems of thermoelasticity were considered for two-component mixtures. 
In the present paper, we study a nonclassical boundary value problem of oscillation of two-component elastic mixtures with spherical cavity. A uniqueness theorem is proved. Solutions of the problem are obtained in terms of absolutely and uniformly convergent series.

\section{Basic Equations}

Let $R^{3}$ be a three-component Euclidean space, and $\Omega^{+} \subset R^{3}$ be a finite domain bounded by the surface $\partial \Omega, \bar{\Omega}^{+}=\Omega^{+} \cup \partial \Omega, \Omega^{-}=R^{3} \backslash \bar{\Omega}^{+}$.

Denote by $u^{\prime}=\left(u_{1}^{\prime}, u_{2}^{\prime}, u_{3}^{\prime}\right)^{\top}$ and $u^{\prime \prime}=\left(u_{1}^{\prime \prime}, u_{2}^{\prime \prime}, u_{3}^{\prime \prime}\right)^{\top}$ the partial displacement vectors; here $\top$ is the transposition symbol. In what follows, under the vector we will mean the one-column matrix.

Homogeneous dynamic equations of two-component elastic mixtures are written in the form [8], [17]

$$
\begin{aligned}
a_{1} \Delta u^{\prime}(x, t) & +b_{1} \operatorname{grad} \operatorname{div} u^{\prime}(x, t)+c \Delta u^{\prime \prime}(x, t) \\
& +d \operatorname{grad} \operatorname{div} u^{\prime \prime}(x, t)=\rho_{1} \frac{\partial^{2} u^{\prime}(x, t)}{\partial t^{2}}, \\
c \Delta u^{\prime}(x, t) & +d \operatorname{grad} \operatorname{div} u^{\prime}(x, t)+a_{2} \Delta u^{\prime \prime}(x, t) \\
& +b_{2} \operatorname{grad} \operatorname{div} u^{\prime \prime}(x, t)=\rho_{2} \frac{\partial^{2} u^{\prime \prime}(x, t)}{\partial t^{2}} .
\end{aligned}
$$

Let us assume that the displacement components are periodic functions of time, i.e., we can write them in the form $u^{\prime}(x, t)=u^{\prime}(x) \exp (-i t \sigma), u^{\prime \prime}(x, t)=$ $u^{\prime \prime}(x) \exp (-i t \sigma)$, where $\sigma \in R^{1}$ and $i=\sqrt{-1}$. Then we obtain the following system of homogeneous differential equations of stationary oscillation of twocomponent elastic mixtures:

$$
\begin{aligned}
a_{1} \Delta u^{\prime}(x) & +b_{1} \operatorname{grad} \operatorname{div} u^{\prime}(x)+c \Delta u^{\prime \prime}(x) \\
& +d \operatorname{grad} \operatorname{div} u^{\prime \prime}(x)+\rho_{1} \sigma^{2} u^{\prime}(x)=0, \\
c \Delta u^{\prime}(x) & +d \operatorname{grad} \operatorname{div} u^{\prime}(x)+a_{2} \Delta u^{\prime \prime}(x) \\
& +b_{2} \operatorname{grad} \operatorname{div} u^{\prime \prime}(x)+\rho_{2} \sigma^{2} u^{\prime \prime}(x)=0
\end{aligned}
$$

where $u^{\prime}=\left(u_{1}^{\prime}, u_{2}^{\prime}, u_{3}^{\prime}\right), u^{\prime \prime}=\left(u_{1}^{\prime \prime}, u_{2}^{\prime \prime}, u_{3}^{\prime \prime}\right)$ are partial displacement vectors; $\rho_{1}, \rho_{2}$ are partial mixture densities, and $\sigma$ is an oscillation frequency,

$$
\begin{gathered}
a_{1}=\mu_{1}-\lambda_{5}, \quad b_{1}=\mu_{1}+\lambda_{5}+\lambda_{1}-\frac{\rho_{2}}{\rho} \alpha^{\prime}, \quad a_{2}=\mu_{2}-\lambda_{5}, \\
b_{2}=\mu_{2}+\lambda_{5}+\lambda_{2}+\frac{\rho_{1}}{\rho} \alpha^{\prime}, \quad c=\mu_{3}+\lambda_{5}, \quad \alpha^{\prime}=\lambda_{3}-\lambda_{4}, \\
d=\mu_{3}-\lambda_{5}+\lambda_{3}-\frac{\rho_{1}}{\rho} \alpha^{\prime}, \quad \rho=\rho_{1}+\rho_{2},
\end{gathered}
$$

$\lambda_{1}, \lambda_{2}, \ldots, \lambda_{5}, \mu_{1}, \mu_{2}, \mu_{3}$ are the elasticity moduli which satisfy the conditions $[17]$

$$
\mu_{1}>0, \quad \mu_{1} \mu_{2}-\mu_{3}^{2}>0, \quad \lambda_{5}<0, \quad \lambda_{1}+\frac{2}{3} \mu_{1}-\frac{\rho_{2}}{\rho} \alpha^{\prime}>0
$$




$$
\left(\lambda_{1}+\frac{2}{3} \mu_{1}-\frac{\rho_{2}}{\rho} \alpha^{\prime}\right)\left(\lambda_{2}+\frac{2}{3} \mu_{2}+\frac{\rho_{1}}{\rho} \alpha^{\prime}\right)>\left(\lambda_{3}+\frac{2}{3} \mu_{3}-\frac{\rho_{1}}{\rho} \alpha^{\prime}\right)^{2} .
$$

From the above inequalities it follows that

$$
d_{1}=\left(a_{1}+b_{1}\right)\left(a_{2}+b_{2}\right)-(c+d)^{2}>0, \quad d_{2}=a_{1} a_{2}-c^{2}>0 .
$$

Definition 2.1. The vector $U=\left(u^{\prime}, u^{\prime \prime}\right)^{\top}$ defined in the domain $\Omega \subset R^{3}$ is called regular if $u_{k}^{\prime}, u_{k}^{\prime \prime} \in C^{2}(\Omega) \cap C^{1}(\bar{\Omega}), k=1,2,3, \bar{\Omega}=\Omega \cup \partial \Omega$.

\section{Expansion of Regular Solutions}

Theorem 3.1. A regular solution $U=\left(u^{\prime}, u^{\prime \prime}\right)^{\top}$ of the homogeneous oscillation equation (2.1) is represented as a sum

$$
u^{\prime}=\sum_{j=1}^{4} \alpha_{j} V_{j}, \quad u^{\prime \prime}=\sum_{j=1}^{4} \beta_{j} V_{j},
$$

where $V_{j}, j=1,2,3,4$, are regular vectors satisfying the conditions

$$
\begin{gathered}
\left(\Delta+k_{j}^{2}\right) V_{j}=0, \quad j=1,2,3,4, \\
\operatorname{rot} V_{j}=0, \quad j=1,2, \quad \operatorname{div} V_{j}=0, \quad j=3,4,
\end{gathered}
$$

and, also,

$$
\begin{gathered}
\alpha_{j}=(c+d) k_{j}^{2}, \quad \beta_{j}=\rho_{1} \sigma^{2}-\left(a_{1}+b_{1}\right) k_{j}^{2}, \quad j=1,2, \\
\alpha_{j}=c k_{j}^{2}, \quad \beta_{j}=\rho_{1} \sigma^{2}-a_{1} k_{j}^{2}, \quad j=3,4 ; \\
k_{1}^{2}+k_{2}^{2}=\frac{1}{d_{1}}\left[\rho_{1}\left(a_{2}+b_{2}\right)+\rho_{2}\left(a_{1}+b_{1}\right)\right] \sigma^{2}, \quad k_{1}^{2} k_{2}^{2}=\frac{\rho_{1} \rho_{2} \sigma^{4}}{d_{1}} \\
k_{3}^{2}+k_{4}^{2}=\frac{1}{d_{2}}\left(\rho_{1} a_{2}+\rho_{2} a_{1}\right) \sigma^{2}, \quad k_{3}^{2} k_{4}^{2}=\frac{\rho_{1} \rho_{2} \sigma^{4}}{d_{2}} .
\end{gathered}
$$

Proof. We rewrite system (2.1) as follows:

$$
u^{\prime}=\sum_{j=1}^{4} \alpha_{j} V_{j}, \quad u^{\prime \prime}=\sum_{j=1}^{4} \beta_{j} V_{j},
$$

where $\alpha_{j}, \beta_{j}, j=1,2,3,4$, are written as (3.4), and

$$
\begin{aligned}
& V_{1}=\frac{1}{(c+d) \rho_{1} \sigma^{2}\left(k_{2}^{2}-k_{1}^{2}\right) k_{1}^{2}} \operatorname{grad}\left(\beta_{2} \operatorname{div} u^{\prime}-\alpha_{2} \operatorname{div} u^{\prime \prime}\right), \\
& V_{2}=\frac{1}{(c+d) \rho_{1} \sigma^{2}\left(k_{2}^{2}-k_{1}^{2}\right) k_{2}^{2}} \operatorname{grad}\left(\alpha_{1} \operatorname{div} u^{\prime \prime}-\beta_{1} \operatorname{div} u^{\prime}\right), \\
& V_{3}=\frac{1}{c \rho_{1} \sigma^{2}\left(k_{3}^{2}-k_{4}^{2}\right) k_{3}^{2}} \operatorname{rot}\left(\beta_{4} \operatorname{rot} u^{\prime}-\alpha_{4} \operatorname{rot} u^{\prime \prime}\right), \\
& V_{4}=\frac{1}{c \rho_{1} \sigma^{2}\left(k_{3}^{2}-k_{4}^{2}\right) k_{4}^{2}} \operatorname{rot}\left(\alpha_{3} \operatorname{rot} u^{\prime \prime}-\beta_{3} \operatorname{rot} u^{\prime}\right),
\end{aligned}
$$

where $k_{j}^{2}, j=1,2,3,4$, are written as (3.5). 
Since rot grad $=0$ and div rot $=0$, from $(3.7)$ we obtain

$$
\operatorname{rot} V_{j}=0, \quad j=1,2, \quad \operatorname{div} V_{j}=0, \quad j=3,4 .
$$

The theorem will be proved, if we show that

$$
\left(\Delta+k_{j}^{2}\right) V_{j}=0, \quad j=1,2,3,4 .
$$

The statement below is true [14].

Theorem 3.2. A regular solution of the system of homogeneous differential equations (2.1) has continuous partial derivatives of any order at an arbitrary point not belonging to $\partial \Omega$.

By Theorem 3.2, to each equation of system (2.1) we can apply the operation div; then, taking into account div grad $\equiv \Delta$, we obtain

$$
\begin{aligned}
& \left(a_{1}+b_{1}\right) \Delta \operatorname{div} u^{\prime}+(c+d) \Delta \operatorname{div} u^{\prime \prime}+\rho_{1} \sigma^{2} \operatorname{div} u^{\prime}=0, \\
& (c+d) \Delta \operatorname{div} u^{\prime}+\left(a_{2}+b_{2}\right) \Delta \operatorname{div} u^{\prime \prime}+\rho_{2} \sigma^{2} \operatorname{div} u^{\prime \prime}=0 .
\end{aligned}
$$

After multiplying the first equation of system (3.8) by $\beta_{2}$, and the second by $-\alpha_{2}$ and summing the products, we have

$$
\begin{gathered}
{\left[\frac{\left(a_{1}+b_{1}\right) \beta_{2}}{\rho_{1} \sigma^{2}}-\frac{(c+d) \alpha_{2}}{\rho_{2} \sigma^{2}}\right] \Delta \operatorname{div} u^{\prime}+\left[\frac{(c+d) \beta_{2}}{\rho_{1} \sigma^{2}}-\frac{\left(a_{2}+b_{2}\right) \alpha_{2}}{\rho_{2} \sigma^{2}}\right] \Delta \operatorname{div} u^{\prime \prime}+} \\
+\left(\beta_{2} \operatorname{div} u^{\prime}-\alpha_{2} \operatorname{div} u^{\prime \prime}\right)=0 .
\end{gathered}
$$

By (3.4) and (3.5) we obtain

$$
\begin{aligned}
& \frac{\left(a_{1}+b_{1}\right) \beta_{2}}{\rho_{1} \sigma^{2}}-\frac{(c+d) \alpha_{2}}{\rho_{2} \sigma^{2}}=\frac{\beta_{2}}{k_{1}^{2}}, \\
& \frac{(c+d) \beta_{2}}{\rho_{1} \sigma^{2}}-\frac{\left(a_{2}+b_{2}\right) \alpha_{2}}{\rho_{2} \sigma^{2}}=-\frac{\alpha_{2}}{k_{1}^{2}} .
\end{aligned}
$$

The substitution of these equalities into (3.9) gives

$$
\left(\Delta+k_{1}^{2}\right)\left(\beta_{2} \operatorname{div} u^{\prime}-\alpha_{2} \operatorname{div} u^{\prime \prime}\right)=0 .
$$

Hence, using the values $v_{1}$ from (3.7) we obtain

$$
\left(\Delta+k_{1}\right) v_{1}=0 .
$$

After multiplying the first equation of system (3.8) by $-\beta_{1}$, and the second by $\alpha_{1}$ and summing the products, we have

$$
\begin{gathered}
{\left[\frac{(c+d) \alpha_{1}}{\rho_{2} \sigma^{2}}-\frac{\left(a_{1}+b_{1}\right) \beta_{1}}{\rho_{1} \sigma^{2}}\right] \Delta \operatorname{div} u^{\prime}+\left[\frac{\left(a_{2}+b_{2}\right) \alpha_{1}}{\rho_{2} \sigma^{2}}-\frac{(c+d) \beta_{1}}{\rho_{1} \sigma^{2}}\right] \Delta \operatorname{div} u^{\prime \prime}+} \\
+\left(\alpha_{1} \operatorname{div} u^{\prime \prime}-\beta_{1} \operatorname{div} u^{\prime}\right)=0 .
\end{gathered}
$$

By (3.4) and (3.5) we obtain

$$
\begin{aligned}
& \frac{(c+d) \alpha_{1}}{\rho_{2} \sigma^{2}}-\frac{\left(a_{1}+b_{1}\right) \beta_{1}}{\rho_{1} \sigma^{2}}=-\frac{\beta_{1}}{k_{2}^{2}}, \\
& \frac{\left(a_{2}+b_{2}\right) \alpha_{1}}{\rho_{2} \sigma^{2}}-\frac{(c+d) \beta_{1}}{\rho_{1} \sigma^{2}}=\frac{\alpha_{1}}{k_{2}^{2}} .
\end{aligned}
$$


The substitution of these equalities into (3.10) gives

$$
\left(\Delta+k_{2}^{2}\right)\left(\alpha_{1} \operatorname{div} u^{\prime \prime}-\beta_{1} \operatorname{div} u^{\prime}\right)=0 .
$$

This means that $\left(\Delta+k_{2}\right) v_{2}=0$.

Applying the operation rot to both parts of each equation of system (2.1), we obtain

$$
\begin{aligned}
& a_{1} \Delta \operatorname{rot} u^{\prime}+c \Delta \operatorname{rot} u^{\prime \prime}+\rho_{1} \sigma^{2} \operatorname{rot} u^{\prime}=0, \\
& c \Delta \operatorname{rot} u^{\prime}+a_{2} \Delta \operatorname{rot} u^{\prime \prime}+\rho_{2} \sigma^{2} \operatorname{rot} u^{\prime \prime}=0 .
\end{aligned}
$$

After multiplying the first equation of system (3.11) by $\beta_{4}$, and the second by $-\alpha_{4}$, we have

$$
\begin{gathered}
\left(\frac{a_{1} \beta_{4}}{\rho_{1} \sigma^{2}}-\frac{c \alpha_{4}}{\rho_{2} \sigma^{2}}\right) \Delta \operatorname{rot} u^{\prime}\left(\frac{c \beta_{4}}{\rho_{1} \sigma^{2}}-\frac{a_{2} \alpha_{4}}{\rho_{2} \sigma^{2}}\right) \Delta \operatorname{div} u^{\prime \prime}+ \\
+\left(\beta_{4} \operatorname{rot} u^{\prime}-\alpha_{4} \operatorname{rot} u^{\prime \prime}\right)=0 .
\end{gathered}
$$

If in these equations we take into account that

$$
\begin{aligned}
& \frac{a_{1} \beta_{4}}{\rho_{1} \sigma^{2}}-\frac{c \alpha_{4}}{\rho_{2} \sigma^{2}}=\frac{\beta_{4}}{k_{3}^{2}}, \\
& \frac{c \beta_{4}}{\rho_{1} \sigma^{2}}-\frac{a_{2} \alpha_{4}}{\rho_{2} \sigma^{2}}=-\frac{\alpha_{4}}{k_{3}^{2}},
\end{aligned}
$$

then we obtain

$$
\left(\Delta+k_{3}^{2}\right)\left(\beta_{4} \operatorname{rot} u^{\prime}-\alpha_{4} \operatorname{rot} u^{\prime \prime}\right)=0
$$

Taking into account the value of the vector $V_{3}$ from (3.7), from the latter equality we obtain $\left(\Delta+k_{3}^{2}\right) V_{3}=0$.

In an analogous manner we prove that $\left(\Delta+k_{4}^{2}\right) V_{4}=0$.

Definition 3.3. A regular in $\Omega^{-}$solution $U=\left(u^{\prime}, u^{\prime \prime}\right)^{\top}$ of the system of homogeneous equations (2.1) satisfy the radiation condition if for the vectors $V_{j}$ given by equalities (3.7) the conditions

$$
\begin{aligned}
V_{j}(x) & =o(1), \\
\frac{\partial V_{j}(x)}{\partial R}-i k_{j} V_{j}(x) & =o\left(R^{-1}\right), \quad j=1,2,3,4,
\end{aligned}
$$

are fulfilled, where $R$ is the length of the radius vector of the point $x=$ $\left(x_{1}, x_{2}, x_{3}\right)$; the derivative with respect to $R$ is a partial derivative with respect to the coordinate $R$ of the point $x . \Omega^{-}=R^{3} \backslash\left(\bar{\Omega}^{+}\right), \Omega^{+}$is a finite domain bounded by the surface $\partial \Omega$.

The theorems below are true [7], [14], [16].

Theorem 3.4. If $U=\left(u^{\prime}, u^{\prime \prime}\right)^{\top}$ is a regular solution of system (2.1) in $\Omega^{-}$ and satisfies the radiation condition, then

$$
V_{j}(x)=O\left(R^{-1}\right), \quad \frac{\partial V_{j}(x)}{\partial R}-i k_{j} V_{j}(x)=O\left(R^{-2}\right), \quad j=1,2,3,4 .
$$


Theorem 3.5. For the vector $u=\left(u_{1}, u_{2}, u_{3}\right)$ to be a solution of the system of differential equations

$$
\left(\Delta+k^{2}\right) u=0, \quad \operatorname{div} u=0,
$$

in the domain $\Omega \subset R^{3}$, it is necessary and sufficient that it be represented in the form

$$
u(x)=\operatorname{rot} \operatorname{rot}\left(x \chi_{1}(x)\right)+\operatorname{rot}\left(x \chi_{2}(x)\right),
$$

where $k$ is a constant, $x=\left(x_{1}, x_{2}, x_{3}\right),\left(\Delta+k^{2}\right) \chi_{j}(x)=0, j=1,2$.

Theorem 3.6. For the vector $u=\left(u_{1}, u_{2}, u_{3}\right)$ to be a solution of the system of differential equations

$$
\left(\Delta+k^{2}\right) u=0, \quad \operatorname{rot} u=0,
$$

in the domain $\Omega \subset R^{3}$, it is necessary and sufficient that it be represented in the form

$$
u(x)=\operatorname{grad} \chi(x)
$$

where $k$ is a constant, $\chi(x)$ is a metaharmonic function, i.e., $\left(\Delta+k^{2}\right) \chi(x)=0$.

Let us prove the following theorem.

Theorem 3.7. For the vector $U=\left(u^{\prime}, u^{\prime \prime}\right)^{\top}$ to be a solution of the system of differential equations (2.1) in the domain $\Omega \subset R^{3}$, it is necessary and sufficient that it be represented in the form

$$
\begin{aligned}
u^{\prime}(x) & =\operatorname{grad}\left[\alpha_{1} \Phi_{1}(x)+\alpha_{2} \Phi_{2}(x)\right]+\operatorname{rot} \operatorname{rot}\left(x \Psi_{3}(x)\right)+\operatorname{rot}\left(x \Psi_{5}(x)\right), \\
u^{\prime \prime}(x) & =\operatorname{grad}\left[\beta_{1} \Phi_{1}(x)+\beta_{2} \Phi_{2}(x)\right]+\operatorname{rot} \operatorname{rot}\left(x \Psi_{4}(x)\right)+\operatorname{rot}\left(x \Psi_{6}(x)\right),
\end{aligned}
$$

where

$$
\begin{aligned}
& \Psi_{2 j+1}(x)=\alpha_{3} \Phi_{2 j+1}(x)+\alpha_{4} \Phi_{2 j+2}(x), \\
& \Psi_{2 j+2}(x)=\beta_{3} \Phi_{2 j+1}(x)+\beta_{4} \Phi_{2 j+2}(x), \quad j=1,2,
\end{aligned}
$$

$\Phi_{j}(x), j=1,2, \ldots, 6$, are scalar metaharmonic functions satisfying the equations

$$
\left(\Delta+k_{j}^{2}\right) \Phi_{j}(x)=0, \quad j=1,2,3,4, \quad\left(\Delta+k_{j}^{2}\right) \Phi_{j+2}(x)=0, \quad j=3,4,
$$

the constants $\alpha_{j}, \beta_{j}, k_{j}^{2}, j=1,2,3,4$, have form (3.4), (3.5).

Proof. We will prove the first part of the theorem. Let the vector $U=\left(u^{\prime}, u^{\prime \prime}\right)^{\top}$ be a solution of system (2.1). Then, by virtue of Theorem 3.1, it can be represented as (3.6), where $\alpha_{j}, \beta_{j}, j=1,2,3,4$, have form (3.4), and the vectors $V_{j}, j=1,2,3,4$, satisfy the system of differential equations (3.2), (3.3). The constants $k_{j}^{2}, j=1,2,3,4$, have form (3.5).

By virtue of Theorems 3.5 and 3.6, the vectors $V_{j}, j=1,2,3,4$, can be written in the form

$$
\begin{aligned}
& V_{j}(x)=\operatorname{grad} \Phi_{j}(x), \quad j=1,2, \\
& V_{3}(x)=\operatorname{rot} \operatorname{rot}\left(x \Phi_{3}\right)+\operatorname{rot}\left(x \Phi_{5}\right), \\
& V_{4}(x)=\operatorname{rot} \operatorname{rot}\left(x \Phi_{4}\right)+\operatorname{rot}\left(x \Phi_{6}\right),
\end{aligned}
$$

where the scalar functions $\Phi_{j}(x), j=1,2, \ldots, 6$, satisfy equations (3.13). 
If we substitute the values of the vectors $V_{j}, j=1,2,3,4$, into (3.6), then we obtain representation (3.12). This completes the proof of the first part of the theorem.

Substituting the values of the vectors $u^{\prime}(x)$ and $u^{\prime \prime}(x)$ from (3.12) into (2.1) and using the identities

$$
\begin{aligned}
& \beta_{j}\left[\rho_{2} \sigma^{2}-\left(a_{2}+b_{2}\right) k_{j}^{2}\right]-(c+d) k_{j}^{2} \alpha_{j}=0, \quad j=1,2, \\
& \beta_{j}\left[\rho_{2} \sigma^{2}-a_{2} k_{j}^{2}\right]-c k_{j}^{2} \alpha_{j}=0, \quad j=3,4,
\end{aligned}
$$

we ascertain that the vector $U=\left(u^{\prime}, u^{\prime \prime}\right)^{\top}$ represented by (3.12) is a solution of system (2.1).

\section{Formulation of the Problem. The Uniqueness Theorem}

Denote by $\Omega^{+}$the ball bounded by the spherical surface $\partial \Omega$ with center at the origin and radius $R$, i.e., $\Omega^{+}=\left\{x: x \in R^{3},|x|<R\right\}, \partial \Omega=\left\{x: x \in R^{3}\right.$, $|x|=R\}, \Omega^{-}=R^{3} \backslash \bar{\Omega}^{+}$.

Problem $(\stackrel{\sigma}{N})^{-}$. Find, a regular solution of system $(2.1)$ in the domain $\Omega^{-}$, which on the boundary $\partial \Omega$ satisfies the conditions

$$
\begin{array}{ll}
{\left[n(z) \cdot u^{\prime}(z)\right]^{-}=f_{4}^{(1)}(z),} & {\left[n(z) \cdot u^{\prime \prime}(z)\right]^{-}=f_{4}^{(2)}(z),} \\
{\left[n(z) \times \operatorname{rot} u^{\prime}(z)\right]^{-}=f^{(1)}(z),} & {\left[n(z) \times \operatorname{rot} u^{\prime \prime}(z)\right]^{-}=f^{(2)}(z),}
\end{array}
$$

where $f^{(j)}=\left(f_{1}^{(j)}(z), f_{2}^{(j)}(z), f_{3}^{(j)}(z)\right), j=1,2, f_{k}^{(j)}, j=1,2, k=1,2,3,4$ are the functions given on $\partial \Omega, n(z)$ is the outward unit normal with respect to $\Omega^{+}$ at a point $z \in \partial \Omega$.

Near the point at infinity the vector $U=\left(u^{\prime}, u^{\prime \prime}\right)^{\top}$ satisfies the radiation condition.

We introduce the matrix differential operator $A(\partial x)$

$$
\begin{aligned}
& A(\partial x)=\left[\begin{array}{lll}
A^{(1)}(\partial x) & \vdots & A^{(2)}(\partial x) \\
\ldots \ldots \ldots \ldots \ldots \ldots \ldots \\
A^{(3)}(\partial x) & \vdots & A^{(4)}(\partial x)
\end{array}\right]_{6 \times 6}, \\
& A^{(i)}(\partial x)=\left[A_{k j}^{(i)}(\partial x)\right]_{3 \times 3}, \quad i=1,2,3,4,
\end{aligned}
$$

where

$$
\begin{aligned}
A_{k j}^{(1)} & =a_{1} \delta_{k j} \Delta+b_{1} \frac{\partial^{2}}{\partial x_{k} \partial x_{j}}, \\
A_{k j}^{(i)} & =c \delta_{k j} \Delta+d \frac{\partial^{2}}{\partial x_{k} \partial x_{j}}, \quad i=2,3, \\
A_{k j}^{(4)} & =a_{2} \delta_{k j} \Delta+b_{2} \frac{\partial^{2}}{\partial x_{k} \partial x_{j}}
\end{aligned}
$$

$\delta_{k j}$ is Kronecker's symbol and $\Delta$ the Laplace operator. 

[14].

Denote by $\Omega$ the finite domain bounded by the surface $\partial \Omega \in \Lambda_{1}(\alpha), 0<\alpha \leq 1$

Theorem 4.1. If $U=\left(u^{\prime}, u^{\prime \prime}\right)^{\top}$ is a regular vector in the domain $\Omega$ and $A(\partial x) U$ is absolutely integrable in $\Omega$, then

$$
\int_{\Omega} U \cdot A(\partial x) \bar{U} d x=\int_{\partial \Omega}[U(z)]^{+} \cdot[P(\partial z, n) \bar{U}(z)]^{+} d s-\int_{\Omega} E(U, \bar{U}) d x,
$$

where the small line above the symbol indicates a complex-conjugate value,

$$
\begin{aligned}
U \cdot P \bar{U}= & {\left[\left(a_{1}+b_{1}\right)\left(n \cdot u^{\prime}\right)+(c+d)\left(n \cdot u^{\prime \prime}\right)\right] \operatorname{div} \bar{u}^{\prime} } \\
& +\left[(c+d)\left(n \cdot u^{\prime}\right)+\left(a_{2}+b_{2}\right)\left(n \cdot u^{\prime \prime}\right)\right] \operatorname{div} \bar{u}^{\prime \prime} \\
& -\left(a_{1} u^{\prime}+c u^{\prime \prime}\right) \cdot\left[n \times \operatorname{rot} \bar{u}^{\prime}\right]-\left(c u^{\prime}+a_{2} u^{\prime \prime}\right) \cdot\left[n \times \operatorname{rot} \bar{u}^{\prime \prime}\right] \\
E(U, \bar{U})= & \frac{1}{a_{1}+b_{1}}\left[\left|\left(a_{1}+b_{1}\right) \operatorname{div} u^{\prime}+(c+d) \operatorname{div} u^{\prime \prime}\right|^{2}+d_{1}\left|\operatorname{div} u^{\prime \prime}\right|^{2}\right] \\
& +\frac{1}{a_{1}}\left[\left|a_{1} \operatorname{rot} u^{\prime}+c \operatorname{rot} u^{\prime \prime}\right|^{2}+d_{2}\left|\operatorname{rot} u^{\prime \prime}\right|^{2}\right] .
\end{aligned}
$$

Proof. Let $U=\left(u^{\prime}, u^{\prime \prime}\right)^{\top}$ and $\bar{U}=\left(\bar{u}^{\prime}, \bar{u}^{\prime \prime}\right)^{\top}$ be six-component complex-conjugate vectors. We will consider the scalar derivative

$$
\begin{aligned}
U \cdot A(\partial x) \bar{U}= & \left(a_{1} u^{\prime}+c u^{\prime \prime}\right) \cdot \Delta \bar{u}^{\prime}+\left(c u^{\prime}+a_{2} u^{\prime \prime}\right) \cdot \Delta \bar{u}^{\prime \prime} \\
& +\left(b_{1} u^{\prime}+d u^{\prime \prime}\right) \cdot \operatorname{grad} \operatorname{div} \bar{u}^{\prime}+\left(d u^{\prime}+b_{2} u^{\prime \prime}\right) \cdot \operatorname{grad} \operatorname{div} \bar{u}^{\prime \prime} .
\end{aligned}
$$

After some transformations we obtain

$$
u \cdot \Delta v=\operatorname{div}(u \operatorname{div} v)-\operatorname{div} u \operatorname{div} v+\operatorname{div}[u \times \operatorname{rot} v]-\operatorname{rot} u \cdot \operatorname{rot} v
$$$$
u \cdot \operatorname{grad} \operatorname{div} v=\operatorname{div}(u \operatorname{div} v)-\operatorname{div} u \operatorname{div} v,
$$

where $u=\left(u_{1}, u_{2}, u_{3}\right)^{\top}$ and $v=\left(v_{1}, v_{2}, v_{3}\right)^{\top}$ are three-component vectors.

Substituting these equalities into (4.6), we have

$$
\begin{aligned}
U \cdot A(\partial x) \bar{U}= & \operatorname{div}\left\{\left[\left(a_{1}+b_{1}\right) u^{\prime}+(c+d) u^{\prime \prime}\right] \operatorname{div} \bar{u}^{\prime}+\left[(c+d) u^{\prime}\right.\right. \\
& \left.+\left(a_{2}+b_{2}\right) u^{\prime \prime}\right] \operatorname{div} \bar{u}^{\prime \prime}+a_{1}\left[u^{\prime} \times \operatorname{rot} \bar{u}^{\prime}\right]+c\left[u^{\prime} \times \operatorname{rot} \bar{u}^{\prime \prime}\right] \\
& \left.+c\left[u^{\prime \prime} \times \operatorname{rot} \bar{u}^{\prime}\right]+a_{2}\left[u^{\prime \prime} \times \operatorname{rot} \bar{u}^{\prime \prime}\right]\right\}-E(U, \bar{U}),
\end{aligned}
$$

where $E(U, \bar{U})$ has form (4.5).

Applying the Gauss-Ostrogradski formula, from (4.7) we obtain equality (4.3), where

$$
\begin{aligned}
U \cdot P \bar{U}= & n \cdot\left\{\left[\left(a_{1}+b_{1}\right) u^{\prime}+(c+d) u^{\prime \prime}\right] \operatorname{div} \bar{u}^{\prime}+\left[(c+d) u^{\prime}\right.\right. \\
& \left.+\left(a_{2}+b_{2}\right) u^{\prime \prime}\right] \operatorname{div} \bar{u}^{\prime \prime}+a_{1}\left[u^{\prime} \times \operatorname{rot} \bar{u}^{\prime}\right]+c\left[u^{\prime} \times \operatorname{rot} \bar{u}^{\prime \prime}\right] \\
& \left.+c\left[u^{\prime \prime} \times \operatorname{rot} \bar{u}^{\prime}\right]+a_{2}\left[u^{\prime \prime} \times \operatorname{rot} \bar{u}^{\prime \prime}\right]\right\} .
\end{aligned}
$$

If we use here the identity

$$
n \cdot[u \times \operatorname{rot} v]=-u \cdot[n \times \operatorname{rot} v]
$$

then $U \cdot P \bar{U}$ takes form (4.4). 
Since $E(U, \bar{U})=E(\bar{U}, U),(4.3)$ immediately implies

$$
\begin{aligned}
\int_{\Omega}[U \cdot A(\partial x) \bar{U} & -\bar{U} \cdot A(\partial x) U] d x \\
& \left.=\int_{\partial \Omega}[U(z) \cdot P(\partial z, n) \bar{U}(z)-\bar{U}(z) \cdot P(\partial z, n) U)(z)\right]^{+} d S .
\end{aligned}
$$

Theorem 4.2. If $\partial \Omega \in \Lambda_{1}(\alpha), 0<\alpha \leq 1$, then the homogeneous Problem $(\stackrel{\sigma}{N})_{0}^{-}$has only a trivial solution in the class of regular vectors.

Proof. Denote by $B(0, R)$ the ball bounded by the sphere $S(0, R)$ with center at the origin and radius $R$. Let $\Omega_{R}^{-}=\Omega^{-} \cap B(0, R)$, where $R>0$ is a sufficiently large value such that $\partial \Omega \subset B(0, R)$. Applying (4.7) in the domain $\Omega_{R}^{-}$, we obtain

$$
\begin{gathered}
\int_{\Omega_{R}^{-}}[U \cdot A(\partial x) \bar{U}-\bar{U} \cdot A(\partial x) U] d x \\
=\int_{\partial \Omega}[U(z) \cdot P(\partial z, n) \bar{U}(z)-\bar{U}(z) \cdot P(\partial z, n) U(z)]^{-} d S \\
\quad+\int_{S(0, R)}[U \cdot P(\partial z, n) \bar{U}-\bar{U} \cdot P(\partial z, n) U] d S .
\end{gathered}
$$

Rewrite system (2.1) as

$$
A(\partial x) U+\mathcal{J} \sigma^{2} U=0
$$

where the operator $A(\partial x)$ has form (4.2),

$$
\mathcal{J}=\left[\begin{array}{ccc}
\rho_{1} I & \vdots & 0 \\
\ldots & \ldots & \ldots \\
0 & \vdots & \rho_{2} I
\end{array}\right]_{6 \times 6}, \quad I=\left[\delta_{k j}\right]_{3 \times 3}
$$

By virtue of (4.10), the volume integral in equality (4.9) vanishes an so also does the integral on $\partial \Omega$ due to the boundary conditions of Problem $(\stackrel{\sigma}{N})_{0}^{-}\left(f_{4}^{(j)}=\right.$ $\left.0, f^{(j)}=0, j=1,2\right)$. As a result we obtain

$$
\int_{S(0, R)}[U \cdot P(\partial z, n) \bar{U}-\bar{U} \cdot P(\partial z, n) U] d S=0 .
$$


The following estimates are true [14]:

$$
\begin{aligned}
\operatorname{div} V_{j} & =i k_{j}\left(n \cdot V_{j}\right)+O\left(R^{-2}\right), \quad j=1,2, \\
\operatorname{rot} V_{j} & =i k_{j}\left[n \times V_{j}\right]+O\left(R^{-2}\right), \quad j=3,4, \\
\left(n \cdot V_{j}\right) & =O\left(R^{-2}\right), \quad j=3,4, \\
{\left[n \times V_{j}\right] } & =O\left(R^{-2}\right), \quad j=1,2, \\
\left(V_{k} \cdot \bar{V}_{j}\right) & =O\left(R^{-3}\right), \quad\left(\bar{V}_{k} \cdot V_{j}\right)=O\left(R^{-3}\right), \quad k=1,2, \quad j=3,4 .
\end{aligned}
$$

Using the vector identity

$$
[a \times[b \times c]]=b(a \cdot c)-c(a \cdot b),
$$

from the second equality of (4.12) we have

$$
\left[n \times \operatorname{rot} V_{j}\right]=-i k_{j} V_{j}+O\left(R^{-2}\right), \quad j=3,4,
$$

where $n(z)$ is the outward unit normal with respect to $B(0, R)$ at a point $z \in$ $S(0, R)$, the functions $V_{j}$ satisfy equations (3.2) and (3.3).

If to (4.4) we apply formulas (3.1), the radiation condition and estimates (4.12), (4.13), then we obtain

$$
\begin{aligned}
U \cdot P \bar{U}-\bar{U} \cdot P U= & -2 i\left[k_{1} \lambda_{1}^{2}\left|\left(n \cdot V_{1}\right)\right|^{2}+k_{2} \lambda_{2}^{2}\left|\left(n \cdot V_{2}\right)\right|^{2}\right. \\
& \left.+k_{3} \lambda_{3}^{2}\left|V_{3}\right|^{2}+k_{4} \lambda_{4}^{2}\left|V_{4}\right|^{2}\right]+O\left(R^{-3}\right)
\end{aligned}
$$

where

$$
\begin{aligned}
& \lambda_{1}^{2}=\frac{1}{a_{1}+b_{1}}\left\{\left[\alpha_{1}\left(a_{1}+b_{1}\right)+\beta_{1}(c+d)\right]^{2}+\beta_{1}^{2} d_{1}\right\}>0 \\
& \lambda_{2}^{2}=\frac{1}{a_{1}+b_{1}}\left\{\left[\alpha_{2}\left(a_{1}+b_{1}\right)+\beta_{2}(c+d)\right]^{2}+\beta_{2}^{2} d_{1}\right\}>0 \\
& \left.\lambda_{3}^{2}=\frac{1}{a_{1}}\left[\left(\alpha_{3} a_{1}+\beta_{3} c\right)^{2}+\beta_{3}^{2} d_{2}\right)\right]>0 \\
& \left.\lambda_{4}^{2}=\frac{1}{a_{1}}\left[\left(\alpha_{4} a_{1}+\beta_{4} c\right)^{2}+\beta_{4}^{2} d_{2}\right)\right]>0
\end{aligned}
$$

In deriving (4.14), the following identities have been used:

$$
\begin{aligned}
& \alpha_{2}\left[\alpha_{1}\left(a_{1}+b_{1}\right)+\beta_{1}(c+d)\right]+\beta_{2}\left[\alpha_{1}(c+d)+\beta_{1}\left(a_{2}+b_{2}\right)\right]=0, \\
& \alpha_{1}\left[\alpha_{2}\left(a_{1}+b_{1}\right)+\beta_{2}(c+d)\right]+\beta_{1}\left[\alpha_{2}(c+d)+\beta_{2}\left(a_{2}+b_{2}\right)\right]=0, \\
& \alpha_{4}\left(\alpha_{3} a_{1}+\beta_{3} c\right)+\beta_{4}\left(\alpha_{3} c+\beta_{3} a_{2}\right)=0, \\
& \alpha_{3}\left(\alpha_{4} a_{1}+\beta_{4} c\right)+\beta_{3}\left(\alpha_{4} c+\beta_{4} a_{2}\right)=0 .
\end{aligned}
$$

Using estimates (4.14) in (4.11), we obtain

$$
\begin{aligned}
\left.\int_{S(0, R)}\left[k_{1} \lambda_{1}^{2} \mid n \cdot V_{1}\right)\right|^{2} & +k_{2} \lambda_{2}^{2}\left|n \cdot V_{2}\right|^{2} \\
& \left.+k_{3} \lambda_{3}^{2}\left|V_{3}\right|^{2}+k_{3} \lambda_{4}^{2}\left|V_{4}\right|^{2}\right] d S=O\left(R^{-1}\right) .
\end{aligned}
$$


Passing to the limit in (4.15) as $R \rightarrow \infty$, we have

$$
\begin{aligned}
& \lim _{R \rightarrow \infty} \int_{S(0, R)}\left|\left(n \cdot V_{j}\right)\right|^{2} d S=0, \quad i=1,2, \\
& \lim _{R \rightarrow \infty} \int_{S(0, R)}\left|V_{j}\right|^{2} d S=0, \quad i=3,4 .
\end{aligned}
$$

The next lemma holds true [6], [14], [18], [21].

Lemma 4.3. A regular in $\Omega^{-}$solution of the equation $\left(\Delta+k^{2}\right) u=0, k^{2}>0$, satisfying the radiation condition and the condition

$$
\lim _{R \rightarrow \infty} \int_{S(0, R)}|u(x)|^{2} d_{x} S=0
$$

is identically zero.

This lemma was proved by several authors (Rellich [18], Freudenthal [6], I. Vekua [22], Kupradze [14]).

We will prove the following statement.

Lemma 4.4. A regular in $\Omega^{-}$solution of the equation $\left(\Delta+\lambda^{2}\right) V=0$, $\operatorname{rot} V=0, \lambda^{2}>0$, satisfying the radiation condition and the condition

$$
\lim _{R \rightarrow \infty} \int_{S(0, R)}|n \cdot V(x)|^{2} d_{x} S=0
$$

is identically zero.

Proof. By virtue of Theorem 3.6, the vector $V(x)$ can be written as

$$
V(x)=\operatorname{grad} \Phi(x), \quad\left(\Delta+\lambda^{2}\right) \Phi(x)=0 .
$$

Let $(R, \vartheta, \varphi)$ be the spherical coordinates of a point $x \in S(0, R)$. Then the metaharmonic function $\Phi(x)$ can be written in the form [19]

$$
\Phi(x)=\sum_{k=0}^{\infty} \sum_{m=-k}^{k} \frac{1}{\sqrt{R}} H_{k+\frac{1}{2}}^{(1)}(\lambda R) Y_{k}^{(m)}(\vartheta, \varphi) A_{m k},
$$

where $H_{k+\frac{1}{2}}^{(1)}(\lambda R)$ is a Hankel function of first kind, $A_{m k}$ is an arbitrary constant, $Y_{k}^{(m)}(\vartheta, \varphi)$ has the form

$$
Y_{k}^{(m)}(\vartheta, \varphi)=\sqrt{\frac{2 k+1}{4 \pi} \cdot \frac{(k-m) !}{(k+m) !}} P_{k}^{(m)}(\cos \vartheta) e^{i m \varphi},
$$

$P_{k}^{(m)}(\cos \vartheta)$ is an adjoint Legendre function of first kind, $k$-th power and $m$-th order [19]. We have

$$
n(x) \cdot V(x)=\frac{d}{d R} \Phi(x)=\sum_{k=0}^{\infty} \sum_{m=-k}^{k} \frac{d}{d R}\left(\frac{1}{\sqrt{R}} H_{k+\frac{1}{2}}^{(1)}(\lambda R)\right) Y_{k}^{(m)}(\vartheta, \varphi) A_{m k} .
$$


From the properties of completeness and orthogonality of spherical functions it follows by Parceval's theorem that

$$
\int_{0}^{2 \pi} \int_{0}^{\pi}|R(n \cdot V)|^{2} \sin \vartheta d \vartheta d \varphi=\sum_{k=0}^{\infty} \sum_{m=-k}^{k}\left|R \frac{d}{d R}\left(\frac{1}{\sqrt{R}} H_{k+\frac{1}{2}}^{(1)}(\lambda R)\right) A_{m k}\right|^{2}
$$

while condition (4.17) implies that

$$
\lim _{R \rightarrow \infty} \sum_{k=0}^{\infty} \sum_{m=-k}^{k}\left|R \frac{d}{d R}\left(\frac{1}{\sqrt{R}} H_{k+\frac{1}{2}}^{(1)}(\lambda R)\right) A_{m k}\right|^{2}=0
$$

and for each fixed $k$

$$
\lim _{R \rightarrow \infty} R \frac{d}{d R}\left[\frac{1}{\sqrt{R}} H_{k+\frac{1}{2}}^{(1)}(\lambda R)\right] A_{m k}=0 .
$$

When a real argument $x>0$ has large values, the following asymptotic formula is fulfilled for the Hankel function [19]:

$$
H_{\nu}^{(1)}(x)=\sqrt{\frac{2}{\pi x}} e^{i\left(x-\frac{\pi}{2} \nu-\frac{\pi}{4}\right)}+O\left(x^{-\frac{3}{2}}\right) .
$$

This asymptotic formula clearly implies that (4.19) holds true only for $A_{m k}=$ 0 . This means $V(x)=0$.

Using Lemma 4.3 and 4.4, from (4.16) we obtain $V_{j}(x)=0, j=1,2,3,4$, $x \in \Omega^{-}$. Using the latter result in (3.1), we obtain $u^{\prime}(x)=0, u^{\prime \prime}(x)=0$, $x \in \Omega^{-}$. This completes the proof of Theorem 4.2.

\section{Solution of Problem $(\stackrel{\sigma}{N})^{-}$}

A solution of Problem $(\stackrel{\sigma}{N})^{-}$will be sought for in form (3.12), where the functions $\Phi_{j}(x), j=1, \ldots, 6$, are represented as

$$
\begin{gathered}
\Phi_{j}(x)=\sum_{k=0}^{\infty} \sum_{m=-k}^{k} h_{k}\left(k_{j} r\right) Y_{k}^{(m)}(\vartheta, \varphi) A_{m k}^{(j)}, \quad j=1,2,3,4, \\
\Phi_{j+2}(x)=\sum_{k=0}^{\infty} \sum_{m=-k}^{k} h_{k}\left(k_{j} r\right) Y_{k}^{(m)}(\vartheta, \varphi) A_{m k}^{(j+2)}, \quad j=3,4,
\end{gathered}
$$

where $A_{m k}^{(j)}, j=1, \ldots, 6$, are the unknown constants, $Y_{k}^{(m)}(\vartheta, \varphi)$ has form (4.18), $k_{j}, j=1,2,3,4$, have form (3.5),

$$
h_{k}\left(k_{j} r\right)=\sqrt{\frac{R}{r}} \frac{H_{k+\frac{1}{2}}^{(1)}\left(k_{j} r\right)}{H_{k+\frac{1}{2}}^{(1)}\left(k_{j} R\right)} .
$$

We require of the functions $\Phi_{j}(x), j=3,4,5,6$, to satisfy the conditions

$$
\int_{\partial \Omega_{1}} \Phi_{j}(x) d S=0, \quad j=3,4,5,6
$$


where $\Omega_{1}$ is a sphere with center at the origin and radius $R_{1}\left(R<R_{1}<+\infty\right)$.

If we substitute the values of the function $\Phi_{j}(x), j=3,4,5,6$, from (5.1) into (5.2) and use the equality

$$
\int_{\partial \Omega_{1}} Y_{k}^{(m)}(\vartheta, \varphi) d S= \begin{cases}2 \sqrt{\pi} R_{1}^{2} & \text { for } k=m=0 \\ 0 & \text { for other values of } k \text { and } m\end{cases}
$$

then we obtain $A_{00}^{(j)}=0, j=3,4,5,6$.

If we substitute the values of the function $\Phi_{j}(x), j=1, \ldots, 6$, from (5.1) into (3.12) and use the identity [7]

$$
\begin{aligned}
\operatorname{grad}\left[a(r) Y_{k}^{(m)}(\vartheta, \varphi)\right]= & \frac{d a(r)}{d r} X_{m k}(\vartheta, \varphi)+\frac{\sqrt{k(k+1)}}{r} a(r) Y_{m k}(\vartheta, \varphi), \\
\operatorname{rot}\left[x a(r) Y_{k}^{(m)}(\vartheta, \varphi)\right]= & \sqrt{k(k+1)} a(r) Z_{m k}(\vartheta, \varphi), \\
\operatorname{rot} \operatorname{rot}\left[x a(r) Y_{k}^{(m)}(\vartheta, \varphi)\right]= & \frac{k(k+1)}{r} a(r) X_{m k}(\vartheta, \varphi) \\
& +\sqrt{k(k+1)}\left(\frac{d}{d r}+\frac{1}{r}\right) a(r) Y_{m k}(\vartheta, \varphi),
\end{aligned}
$$

where $a(r)$ is a function of $r$,

$$
\begin{aligned}
& X_{m k}(\vartheta, \varphi)=e_{r} Y_{k}^{(m)}(\vartheta, \varphi), \quad k \geq 0, \\
& Y_{m k}(\vartheta, \varphi)=\frac{1}{\sqrt{k(k+1)}}\left(e_{\vartheta} \frac{\partial}{\partial \vartheta}+\frac{e_{\varphi}}{\sin \vartheta} \frac{\partial}{\partial \varphi}\right) Y_{k}^{(m)}(\vartheta, \varphi), \quad k \geq 1, \\
& Z_{m k}(\vartheta, \varphi)=\frac{1}{\sqrt{k(k+1)}}\left(\frac{e_{\vartheta}}{\sin \vartheta} \frac{\partial}{\partial \varphi}-e_{\varphi} \frac{\partial}{\partial \vartheta}\right) Y_{k}^{(m)}(\vartheta, \varphi), \quad k \geq 1,
\end{aligned}
$$

$|m| \leq k, e_{r}, e_{\vartheta}, e_{\varphi}$ are the normal unit vectors

$$
\begin{aligned}
& e_{r}=(\cos \varphi \sin \vartheta, \sin \varphi \sin \vartheta, \cos \vartheta), \\
& e_{\vartheta}=(\cos \varphi \cos \vartheta, \sin \varphi \cos \vartheta,-\sin \vartheta), \\
& e_{\varphi}=(-\sin \varphi, \cos \varphi, 0),
\end{aligned}
$$

then we obtain

$$
\begin{gathered}
u^{\prime}(x)=\sum_{k=0}^{\infty} \sum_{m=-k}^{k}\left\{u_{m k}^{(1)}(r) X_{m k}(\vartheta, \varphi)+\sqrt{k(k+1)}\left[v_{m k}^{(1)}(r) Y_{m k}(\vartheta, \varphi)\right.\right. \\
\left.\left.+w_{m k}^{(1)}(r) Z_{m k}(\vartheta, \varphi)\right]\right\} \\
u^{\prime \prime}(x)=\sum_{k=0}^{\infty} \sum_{m=-k}^{k}\left\{u_{m k}^{(2)}(r) X_{m k}(\vartheta, \varphi)+\sqrt{k(k+1)}\left[v_{m k}^{(2)}(r) Y_{m k}(\vartheta, \varphi)\right.\right. \\
\left.\left.+w_{m k}^{(2)}(r) Z_{m k}(\vartheta, \varphi)\right]\right\}
\end{gathered}
$$




$$
\begin{aligned}
& u_{m k}^{(1)}(r)=\sum_{j=1}^{2} \alpha_{j} \frac{d}{d r} h_{k}\left(k_{j} r\right) A_{m k}^{(j)}+\sum_{j=3}^{4} \alpha_{j} \frac{k(k+1)}{r} h_{k}\left(k_{j} r\right) A_{m k}^{(j)}, \quad k \geq 0, \\
& v_{m k}^{(1)}(r)=\sum_{j=1}^{2} \alpha_{j} \frac{1}{r} h_{k}\left(k_{j} r\right) A_{m k}^{(j)}+\sum_{j=3}^{4} \alpha_{j}\left(\frac{d}{d r}+\frac{1}{r}\right) h_{k}\left(k_{j} r\right) A_{m k}^{(j)}, \quad k \geq 1, \\
& u_{m k}^{(2)}(r)=\sum_{j=1}^{2} \beta_{j} \frac{d}{d r} h_{k}\left(k_{j} r\right) A_{m k}^{(j)}+\sum_{j=3}^{4} \beta_{j} \frac{k(k+1)}{r} h_{k}\left(k_{j} r\right) A_{m k}^{(j)}, \quad k \geq 0, \\
& v_{m k}^{(2)}(r)=\sum_{j=1}^{2} \beta_{j} \frac{1}{r} h_{k}\left(k_{j} r\right) A_{m k}^{(j)}+\sum_{j=3}^{4} \beta_{j}\left(\frac{d}{d r}+\frac{1}{r}\right) h_{k}\left(k_{j} r\right) A_{m k}^{(j)}, \quad k \geq 1, \\
& w_{m k}^{(1)}(r)=\sum_{j=3}^{4} \alpha_{j} h_{k}\left(k_{j} r\right) A_{m k}^{(j+2)}, \quad w_{m k}^{(2)}(r)=\sum_{j=3}^{4} \beta_{j} h_{k}\left(k_{j} r\right) A_{m k}^{(j+2)}, \quad k \geq 1 .
\end{aligned}
$$

Note that since $n(x)=e_{r}$, from (5.3) we have

$n(x) \cdot X_{m k}(\vartheta, \varphi)=Y_{k}^{(m)}(\vartheta, \varphi), \quad n(x) \cdot Y_{m k}(\vartheta, \varphi)=0, \quad n(x) \cdot Z_{m k}(\vartheta, \varphi)=0$.

By virtue of the latter equality, from (5.4) we obtain

$$
\begin{aligned}
& n(x) \cdot u^{\prime}(x)=\sum_{k=0}^{\infty} \sum_{m=-k}^{k} u_{m k}^{(1)}(r) Y_{k}^{(m)}(\vartheta, \varphi), \\
& n(x) \cdot u^{\prime \prime}(x)=\sum_{k=0}^{\infty} \sum_{m=-k}^{k} u_{m k}^{(2)}(r) Y_{k}^{(m)}(\vartheta, \varphi) .
\end{aligned}
$$

From (3.12) we have

$$
\begin{aligned}
\operatorname{rot} u^{\prime}(x) & =\operatorname{rot}\left[x\left(\alpha_{3} k_{3}^{2} \Phi_{3}(x)+\alpha_{4} k_{4}^{2} \Phi_{4}(x)\right]+\operatorname{rot} \operatorname{rot}\left(x \Psi_{5}(x)\right),\right. \\
\operatorname{rot} u^{\prime \prime}(x) & =\operatorname{rot}\left[x\left(\beta_{3} k_{3}^{2} \Phi_{3}(x)+\beta_{4} k_{4}^{2} \Phi_{4}(x)\right]+\operatorname{rot} \operatorname{rot}\left(x \Psi_{6}(x)\right) .\right.
\end{aligned}
$$

Substituting the values of the function $\Phi_{j}(x), j=3,4,5,6$, from (5.1) into (5.7) and taking into account that

$$
\left[e_{r} \times X_{m k}\right]=0, \quad\left[e_{r} \times Y_{m k}\right]=-Z_{m k}, \quad\left[e_{r} \times Z_{m k}\right]=Y_{m k},
$$

we obtain

$$
\begin{aligned}
n(x) \times \operatorname{rot} u^{\prime}(x)= & \sum_{k=1}^{\infty} \sum_{m=-k}^{k} \sum_{j=3}^{4} \sqrt{k(k+1)}\left[\alpha_{j} k_{j}^{2} h_{k}\left(k_{j} r\right) A_{m k}^{(j)} Y_{m k}(\vartheta, \varphi)\right. \\
& \left.-\alpha_{j}\left(\frac{d}{d r}+\frac{1}{r}\right) h_{k}\left(k_{j} r\right) A_{m k}^{(j+2)} Z_{m k}(\vartheta, \varphi)\right], \\
n(x) \times \operatorname{rot} u^{\prime \prime}(x)= & \sum_{k=1}^{\infty} \sum_{m=-k}^{k} \sum_{j=3}^{4} \sqrt{k(k+1)}\left[\beta_{j} k_{j}^{2} h_{k}\left(k_{j} r\right) A_{m k}^{(j)} Y_{m k}(\vartheta, \varphi)\right. \\
& \left.-\beta_{j}\left(\frac{d}{d r}+\frac{1}{r}\right) h_{k}\left(k_{j} r\right) A_{m k}^{(j+2)} Z_{m k}(\vartheta, \varphi)\right] .
\end{aligned}
$$


Since on the sphere $S(0,1)$ the sets $\left\{Y_{k}^{(m)}(\vartheta, \varphi)\right\}_{|m| \leq k, k=0, \infty}$ and $\left\{X_{m k}(\vartheta, \varphi)\right.$, $\left.Y_{m k}(\vartheta, \varphi), Z_{m k}(\vartheta, \varphi)\right\}_{|m| \leq k, k=\overline{0, \infty}}$ form a complete orthonormal system in the space $L_{2}$ and provided that the sufficient condition of smoothness is fulfilled, we can represent the function $f_{4}^{(j)}(z)$ and the vector $f^{(j)}(z), j=1,2$, as Fourier series

$$
\begin{aligned}
f_{4}^{(j)}(z) & =\sum_{k=0}^{\infty} \sum_{m=-k}^{k} \alpha_{m k}^{(j)} Y_{k}^{(m)}(\vartheta, \varphi) \\
f^{(j)}(z)= & \sum_{k=0}^{\infty} \sum_{m=-k}^{k}\left\{\widetilde{\alpha}_{m k}^{(j)} X_{m k}(\vartheta, \varphi)+\sqrt{k(k+1)}\left[\beta_{m k}^{(j)} Y_{m k}(\vartheta, \varphi)\right.\right. \\
& \left.\left.\quad+\gamma_{m k}^{(j)} Z_{m k}(\vartheta, \varphi)\right]\right\}, \quad j=1,2,
\end{aligned}
$$

where $\widetilde{\alpha}_{m k}^{(j)}, \alpha_{m k}^{(j)}, \beta_{m k}^{(j)}, \gamma_{m k}^{(j)}$ are Fourier coefficients.

Taking into account that $n(z) \cdot f^{(j)}(z)=0, j=1,2$, from (5.10) we obtain

$$
f^{(j)}(z)=\sum_{k=1}^{\infty} \sum_{m=-k}^{k} \sqrt{k(k+1)}\left[\beta_{m k}^{(j)} Y_{m k}(\vartheta, \varphi)+\gamma_{m k}^{(j)} Z_{m k}(\vartheta, \varphi)\right], \quad j=1,2 .
$$

Passing on both sides of equalities (5.6) and (5.8) to the limit as $x \rightarrow z \in \partial \Omega$ and taking into account the boundary condition of Problem $(\stackrel{\sigma}{N})^{-}$and also formulas (5.9) and (5.11), for the unknown constants $A_{m k}^{(j)}, j=1, \ldots, 6$, we obtain the following systems of algebraic equations:

$$
\begin{gathered}
\sum_{j=1}^{2} \alpha_{j} \frac{d}{d R} h_{0}\left(k_{j} R\right) A_{00}^{(j)}=\alpha_{00}^{(1)} \\
\sum_{j=1}^{2} \beta_{j} \frac{d}{d R} h_{0}\left(k_{j} R\right) A_{00}^{(j)}=\alpha_{00}^{(2)} \\
\sum_{j=1}^{2} \alpha_{j} \frac{d}{d R} h_{k}\left(k_{j} R\right) A_{m k}^{(j)}+\sum_{j=3}^{4} \alpha_{j} \frac{k(k+1)}{R} A_{m k}^{(j)}=\alpha_{m k}^{(1)}, \\
\sum_{j=1}^{2} \beta_{j} \frac{d}{d R} h_{k}\left(k_{j} R\right) A_{m k}^{(j)}+\sum_{j=3}^{4} \beta_{j} \frac{k(k+1)}{R} A_{m k}^{(j)}=\alpha_{m k}^{(2)}, \\
\sum_{j=3}^{4} \alpha_{j} k_{j}^{2} A_{m k}^{(j)}=\beta_{m k}^{(1)}, \quad \sum_{j=3}^{4} \beta_{j} k_{j}^{2} A_{m k}^{(j)}=\beta_{m k}^{(2)}, \\
\sum_{j=3}^{4} \alpha_{j}\left(\frac{d}{d R}+\frac{1}{R}\right) h_{k}\left(k_{j} R\right) A_{m k}^{(j+2)}=\gamma_{m k}^{(1)}, \\
\sum_{j=3}^{4} \beta_{j}\left(\frac{d}{d R}+\frac{1}{R}\right) h_{k}\left(k_{j} R\right) A_{m k}^{(j+2)}=\gamma_{m k}^{(2)}, \quad k \geq 1 .
\end{gathered}
$$


These systems are compatible by virtue of Theorem 4.2. Their solution has the form

$$
\begin{aligned}
& A_{00}^{(1)}=\frac{1}{h_{0}^{\prime}\left(k_{1} R\right) \Delta_{0}}\left(\beta_{2} \alpha_{00}^{(1)}-\alpha_{2} \alpha_{00}^{(2)}\right) \\
& A_{00}^{(2)}=\frac{1}{h_{0}^{\prime}\left(k_{2} R\right) \Delta_{0}}\left(\alpha_{1} \alpha_{00}^{(2)}-\beta_{1} \alpha_{00}^{(1)}\right) \\
& A_{m k}^{(1)}=\frac{1}{h_{k}^{\prime}\left(k_{1} R\right) \Delta_{0}}\left(\beta_{2} \delta_{m k}^{(1)}-\alpha_{2} \delta_{m k}^{(2)}\right) \\
& A_{m k}^{(2)}=\frac{1}{h_{k}^{\prime}\left(k_{2} R\right) \Delta_{0}}\left(\alpha_{1} \delta_{m k}^{(2)}-\beta_{1} \delta_{m k}^{(1)}\right) \\
& A_{m k}^{(3)}=\frac{1}{k_{3}^{2} \Delta_{1}}\left(\beta_{4} \beta_{m k}^{(1)}-\alpha_{4} \beta_{m k}^{(2)}\right) \\
& A_{m k}^{(4)}=\frac{1}{k_{4}^{2} \Delta_{1}}\left(\alpha_{3} \beta_{m k}^{(2)}-\beta_{3} \beta_{m k}^{(1)}\right) \\
& A_{m k}^{(5)}=\frac{R}{\left(R h_{k}^{\prime}\left(k_{3} R\right)+1\right) \Delta_{1}}\left(\beta_{4} \gamma_{m k}^{(1)}-\alpha_{4} \gamma_{m k}^{(2)}\right) \\
& A_{m k}^{(6)}=\frac{R}{\left(R h_{k}^{\prime}\left(k_{4} R\right)+1\right) \Delta_{1}}\left(\alpha_{3} \gamma_{m k}^{(2)}-\beta_{3} \gamma_{m k}^{(1)}\right)
\end{aligned}
$$

where

$$
\begin{aligned}
\delta_{m k}^{(1)} & =\alpha_{m k}^{(1)}-\frac{k(k+1)}{\rho_{1} \sigma^{2} R}\left(a_{1} \beta_{m k}^{(1)}+c \beta_{m k}^{(2)}\right), \\
\delta_{m k}^{(2)} & =\alpha_{m k}^{(2)}-\frac{k(k+1)}{\rho_{2} \sigma^{2} R}\left(c \beta_{m k}^{(1)}+a_{2} \beta_{m k}^{(2)}\right), \\
\Delta_{0} & =(c+d) \rho_{1} \sigma^{2}\left(k_{1}^{2}-k_{2}^{2}\right), \quad \Delta_{1}=c \rho_{1} \sigma^{2}\left(k_{3}^{2}-k_{4}^{2}\right), \\
h_{k}^{\prime}\left(k_{j} R\right) & =\left.\frac{d}{d r} h_{k}\left(k_{j} r\right)\right|_{r=R} .
\end{aligned}
$$

By substituting the values of the constant $A_{m k}^{(j)}, j=1,2, \ldots, 6$, from (5.14) into (5.4) we obtain a solution of Problem $(\stackrel{\sigma}{N})^{-}$. To justify this solution we have to prove that series (5.4), (5.6) and (5.8) should be absolutely and uniformly convergent in the domain $\bar{\Omega}^{-}$.

The following statement is true [7].

Theorem 5.1. The vectors $X_{m k}(\vartheta, \varphi), Y_{m k}(\vartheta, \varphi), Z_{m k}(\vartheta, \varphi)$ admit the following estimates for any $k \geq 0$ :

$$
\begin{aligned}
& \left|X_{m k}(\vartheta, \varphi)\right| \leq \sqrt{\frac{2 k+1}{4 \pi}}, \quad k \geq 0, \\
& \left|Y_{m k}(\vartheta, \varphi)\right|<\sqrt{\frac{2 k(k+1)}{2 k+1}}, \quad k \geq 1, \\
& \left|Z_{m k}(\vartheta, \varphi)\right|<\sqrt{\frac{2 k(k+1)}{2 k+1}}, \quad k \geq 1 .
\end{aligned}
$$


Moreover, as is known [19],

$$
\left|Y_{k}^{(m)}(\vartheta, \varphi)\right| \leq \sqrt{\frac{2 k+1}{4 \pi}}, \quad k \geq 0 .
$$

If we substitute estimates (5.15) and (5.16) into (5.4) and write Hankel's functions of first kind as $k \rightarrow \infty$ in the form [19]

$$
h_{k}\left(k_{j} r\right) \approx\left(\frac{R}{r}\right)^{k+1}, \quad h_{k}^{\prime}\left(k_{j} r\right) \approx k\left(\frac{R}{r}\right)^{k+1},
$$

then we ascertain that series (5.4) and their partial derivatives of any order are absolutely and uniformly convergent in the domain $\Omega^{-}$.

Let $x \in \partial \Omega$. Then series (5.4) and their partial derivatives of first order will be absolutely and uniformly convergent if we prove the convergence of the majorizing series

$$
\alpha \sum_{k=k_{0}}^{\infty} \sum_{j=1}^{2} k^{\frac{3}{2}}\left[\left|\alpha_{m k}^{(j)}\right|+k^{2}\left|\beta_{m k}^{(j)}\right|+\left|\gamma_{m k}^{(j)}\right|\right]
$$

where $\alpha$ is a positive constant not depending on $k$.

Series (5.17) converges if we require of the coefficients $\alpha_{m k}^{(j)}, \beta_{m k}^{(j)}, \gamma_{m k}^{(j)}, j=1,2$, to have order

$$
\alpha_{m k}^{(j)}=O\left(k^{-3}\right), \quad \beta_{m k}^{(j)}=O\left(k^{-5}\right), \quad \gamma_{m k}^{(j)}=O\left(k^{-3}\right) .
$$

The following theorem is true [7].

Theorem 5.2. If $f^{(j)} \in C^{l}(\partial \Omega), j=1,2$, then the coefficients $\widetilde{\alpha}_{m k}^{(j)}, \beta_{m k}^{(j)}$, $\gamma_{m k}^{(j)}$ admit the following estimates:

$$
\widetilde{\alpha}_{m k}^{(j)}=O\left(k^{-l}\right), \quad \beta_{m k}^{(j)}=O\left(k^{-l-1}\right), \quad \gamma_{m k}^{(j)}=O\left(k^{-l-1}\right), \quad l \geq 1 .
$$

Analogously, if $f_{4}^{(j)} \in C^{l}(\partial \Omega), j=1,2$, then the estimate

$$
\alpha_{m k}^{(j)}=O\left(k^{-l}\right), \quad j=1,2,
$$

is true $[15]$.

With these properties of Fourier coefficients taken into account, we conclude that the coefficients $\alpha_{m k}^{(j)}, \beta_{m k}^{(j)}, \gamma_{m k}^{(j)}, j=1,2$, admit estimates (5.18) if the boundary vector functions satisfy the following boundary conditions:

$$
f_{4}^{(j)} \in C^{3}(\partial \Omega), \quad f^{(j)} \in C^{4}(\partial \Omega), \quad j=1,2 .
$$

Writing Hankel's function of first kind as $r \rightarrow \infty$ in the form [19]

$$
h_{k}\left(k_{j} r\right)=O\left(r^{-1}\right), \quad\left(\frac{d}{d r}-i k_{j}\right) h_{k}\left(k_{j} r\right)=O\left(r^{-2}\right), \quad j=1,2,3,4,
$$

we ascertain that the vectors $u^{\prime}(x)$ and $u^{\prime \prime}(x)$ defined by (5.4) satisfy the radiation condition near the point at infinity. 
Passing on both sides of equalities (5.6) and (5.8) to the limit as $x \rightarrow z \in \partial \Omega$ and using formulas (5.12), (5.13) and (5.9), we obtain

$$
\begin{array}{ll}
{\left[n(z) \cdot u^{\prime}(z)\right]^{-}=f_{4}^{(1)}(z),} & {\left[n(z) \times \operatorname{rot} u^{\prime}(z)\right]^{-}=f^{(1)}(z),} \\
{\left[n(z) \cdot u^{\prime \prime}(z)\right]^{-}=f_{4}^{(2)}(z),} & {\left[n(z) \times \operatorname{rot} u^{\prime \prime}(z)\right]^{-}=f^{(2)}(z), \quad z \in \partial \Omega .}
\end{array}
$$

Thus, for $f_{4}^{(j)} \in C^{3}(\partial \Omega)$ and $f^{(j)} \in C^{4}(\partial \Omega)$, the vector $U=\left(u^{\prime}, u^{\prime \prime}\right)^{\top}$ represented by (5.4) is a solution of Problem $(\stackrel{\sigma}{N})^{-}$.

\section{REFERENCES}

1. M. Aron, On the existence and uniqueness of solutions in the linear theory of mixtures of two elastic solids. Arch. Mech. (Arch. Mech. Stos.) 26(1974), 717-728.

2. R. J. Aтkin, Constitutive theory for a mixture of an isotropic elastic solid and a nonNewtonian fluid. Z. Angew. Math. Phys. 18(1967), 803-825.

3. R. J. Atkin, P. Chadwick, and T. R. Steel, Uniqueness theorems for linearized theories of interacting continua. Mathematika 14(1967), 27-42.

4. A. Borrelli and M. C. Patria, Uniqueness in the boundary value problems for the static equilibrium equations of a mixture of two elastic solids occupying an unbounded domain. Internat. J. Engrg. Sci. 22(1984), No. 1, 23-38.

5. M. J. Crochet and P. M. NAghdi, On constitutive equations for flow of fluid through an elastic solid. Internat. J. Engrg. Sci. 4 (1966), 383-401.

6. H. M. Freudenthal, Über ein Beugungsproblem aus der elektromagnetischen Lichttheorie. Compositio Math. 6(1938), 221-227.

7. L. Giorgashvili, Solution of the basic boundary value problems of stationary thermoelastic oscillations for domains bounded by spherical surfaces. Georgian Math. J. 4(1997), No. 5, 421-438.

8. A. E. Green and T. R. Steel, Constitutive equations for interacting continua. Int. J. Eng. Sci. 4(1966), No. 4, 483-500.

9. A. E. Green and P. M. NAGhdi, On basic equations for mixtures. Quart. J. Mech. Appl. Math. 22(1969), 427-438.

10. A. E. Green and P. M. NAGhdi, On thermodynamics and the nature of the second law for mixtures of interacting continua. Quart. J. Mech. Appl. Math. 31(1978), No. 3, 265-293.

11. R. Hill, Continuum micro-mechanics of elastoplastic polycrystals. J. Mech. Phys. Solids 13(1965), 89-101.

12. L. P. Khoroshun and N. S. Soltanov, Thermoelasticity of two-component mixtures. (Russian) Naukova Dumka, Kiev, 1984.

13. R. J. Knops and T. R. Steel, Uniqueness in the linear theory of a mixture of two elastic solids. Internat. J. Engrg. Sci. 7(1969), 571-577.

14. V. D. Kupradze, T. G. Gegelia, M. O. Basheleishvili, and T. V. BurchuLADZE, Three-dimensional problems of the mathematical theory of elasticity and thermoelasticity. Translated from the second Russian edition. Edited by V. D. Kupradze. North-Holland Series in Applied Mathematics and Mechanics, 25. North-Holland Publishing Co., Amsterdam-New York, 1979.

15. S. G. Mikhlin, On the theory of multidimensional singular integral equations. (Russian) Vestnik Leningr. Univ. 11(1956), No. 1, 3-24. 
16. F. M. Mors and G. Feshbah, Methods of theoretical physics. (Traslated into Russian), v. 2, Izdat. Inostr, Literaturi, Moscow, 1960.

17. D. G. Natroshvili, A. J. Dzhagmaidze, and M. G. Svanadze, Some problems in the linear theory of elastic mixtures. (Russian) Tbilis. Gos. Univ., Tbilisi, 1986.

18. F. RelLich, Über das asymptotische Verhalten der Lösungen von $\Delta u+\lambda u=0$ in unendlichen Gebieten. Jber. Deutsch. Math. Verein. 53(1943). 57-65.

19. A. N. Tikhonov and A. A. SAmarskr, Equations of mathematical physics. (Russian) Nauka, Moscow, 1966.

20. C. Truesdell and R. Toupin, The classical field theories. With an appendix on tensor fields by J. L. Ericksen. Handbuch der Physik, Bd. III/1, 226-793; appendix, 794-858; Springer, Berlin, 1960.

21. A. F. Ulitko, The method of eigenvector functions in three-dimensional problems of the theory of elasticity. (Russian) Naukova Dumka, Kiev, 1979.

22. I. N. Vekua, On multiharmonic functions. (Russian) Trudy Tbilis. Math. Inst. Razmadze 12(1943), 105-174.

(Received 21.10.2004)

Authors' address:

Higher Mathematics Chair \# 99

Georgian Technical University

77, M. Kostava St, Tbilisi 0175

Georgia

E-mail: lgiorgashvili@yahoo.com 